\title{
In What Sense is the Kolmogorov-Sinai Entropy a Measure for Chaotic Behaviour? - Bridging the Gap Between Dynamical Systems Theory and Communication Theory*
}

\author{
ROMAN FRIGG \\ London School of Economics \\ r.p.frigg@lse.ac.uk
}

May 2003

\begin{abstract}
On an influential account, chaos is explained in terms of random behaviour; and random behaviour in turn is explained in terms of having positive Kolmogorov-Sinai entropy (KSE). Though intuitively plausible, the association of the KSE with random behaviour needs justification since the definition of the KSE does not make reference to any notion that is connected to randomness. I provide this justification for the case of Hamiltonian systems by proving that the KSE is equivalent to a generalized version of Shannon's communicationtheoretic entropy under certain plausible assumptions. I then discuss consequences of this equivalence for randomness in chaotic dynamical systems.
\end{abstract}

*Published in British Journal for the Philosophy of Science 55, 2004, 411-434. The PDF file of the article is available at http://www.romanfrigg.org/writings.htm. 


\section{Introduction}

For many years, chaos theory has been a hotly debated topic and its methods have been used to model a great variety of different situations. However, there is still controversy over the proper characterization of chaotic behaviour. Numerous criteria and indicators for the onset of chaos have been suggested, but none of these has gained the status of a 'canonical definition'. Intuitively, chaos has two faces: Random behaviour and sensitive dependence on initial conditions. Accordingly, the great majority of proposals for characterizing chaos fall into one of the following two groups. The first group focuses on the seemingly random, stochastic, unpredictable or haphazard time evolution of chaotic systems and consequently tries to flesh out chaos in terms of randomness. The second group focuses on sensitive dependence on initial conditions. This leads to a study of the properties of trajectories in phase space, in particular their exponential instability: A slight variation in initial conditions produces significant changes in the long term behaviour of trajectories.

This paper is concerned with the first group of proposals. The problem with this type of suggestions is that to characterize a system's behaviour as random or stochastic is not very illuminating since these notions are as much in need of analysis as chaos itself. What does it mean to say that a dynamical system exhibits random behaviour?

Common physical (as well as philosophical) wisdom has it that ergodic theory fits the bill. More specifically, the claim is that the ergodic hierarchy provides a set of concepts which allows for an adequate characterization of random behaviour (see Lichtenberg and Liebermann 1991, 302-12; Ott 1993, 261-2; Reichl 1992, 47-53; Schuster 1988, 203-7; Tabor 1989, 167-74, to mention just a few). A discussion of the entire hierarchy is beyond the scope of this paper, and for the present purpose only one ergodic notion is of importance, the Kolomogorov-Sinai Entropy (KSE, for short). This notion is crucial because it is generally assumed that the move from zero to positive KSE marks the transition from regular to chaotic behaviour. More precisely, the claim is that having positive KSE is a sufficient condition for chaos (Belot and Earman 1997, 155).

Although, at first glance, this might seem plausible, a closer look at the definition of the KSE casts doubt on the legitimacy of its use as a criterion for the presence of chaos. The definition is phrased in terms of the measure of subsets of the phase space and their time evolution, and does not make 
reference to any notion that is connected to randomness. How can such a notion be an indicator for random behaviour?

Three suggestions have been made how to bridge this gap. First, connect the KSE to sensitive dependence on initial conditions (and thereby de facto reduce it to the second group of proposals), second, take algorithmic complexity to be a measure for randomness and relate the KSE to this notion and, third, establish a link between the KSE and the information theoretic notion of entropy. Among these options the third is the most widely used; the notion of information is almost habitually invoked when the interpretation of the KSE as measure for random behaviour is discussed. Ironically, despite its frequent use and its undoubted attractiveness, it is the only one of these three proposals that has no theoretical grounding. In the first case, Pessin's theorem establishes a neat connection between the Lyapunov exponents measuring the divergence of nearby trajectories and the KSE. (Roughly speaking, the theorem says that the KSE equals the sum over the positive Lyapunov exponents.) In the second case, Brudno's theorem can be invoked which basically says that if the phase space satisfies certain (unproblematic) conditions then the KSE is equal to the algorithmic complexity of almost all trajectories.

Surprisingly, there is no theorem which connects the KSE to the information theoretic notion of entropy in roughly the same way Pessin's and Brudno's theorems link the KSE with Lyapunov exponents and algorithmic complexity, respectively. Mathematically minded authors either do not discuss the relationship between the KSE and communication theory at all (Arnold and Avez 1968, Ch.2; Cornfeld et. al 1982, Ch. $10 \S 6$; Rudolph 1990, Ch. 5; Sinai et at. 1980, Ch. 3); or they pay mere lip service to communication theory in that they attach the term 'information' to purely topological notions without elucidating how phase space topologies relate to the conceptual framework of information theory (Eckmann and Ruelle 1985, Sec. 4; Keller 1998, Ch. 3; Nadkarni 1991, 63f.). Others seem to be aware of the fact that there is a gap to bridge, but then content themselves with some rather loose and hand waving remarks, mainly based on lax analogies (Billingsley 1965, Ch. 2; Mañé 1983, Ch. 4; Parry 1981, Ch. 2; Petersen 1983, Ch. 5). ${ }^{1}$ Nowhere in the literature could I find a clear argument connecting entropy in

\footnotetext{
${ }^{1}$ Petersen (1983, 229-34) discusses the problem in some detail; but his arguments are of no help since the expression he gives for the entropy of a source is not correct.
} 
communication theory to its namesake in dynamical system's theory, despite the frequent mention of Shannon.

This raises the question whether the interpretation of the KSE in terms of information can be vindicated. Is there a way to bridge the gap between the topological and the information-theoretic understanding of entropy? The aim of this paper is to provide such an argument for the case of Hamiltonian systems. More specifically, I prove that the KSE is equivalent to the information-theoretic notion of entropy given certain plausible assumptions. But before I embark on this project it seems worthwhile to appreciate what the problems are: First, communication theory and dynamical systems theory work with different conceptual frameworks. The former deals with a finite set of discrete messages and their combinations, while the latter considers a continuous measurable phase space on which an automorphism (a function mapping the phase space onto itself) is defined. Prima facie these two setups bear little, if any, similarity to each other. Second, The mathematical theories are entirely different. The KSE of an automorphism $\Phi$ is defined as $H_{\Phi}=\sup _{\alpha} \lim _{k \rightarrow \infty}(1 / k) H\left(\alpha \vee \Phi \alpha \vee \ldots \vee \Phi^{k-1} \alpha\right)$ and there is not a single formula in communication theory that bears any similarity to this expression. For this reason it is not possible to resort to formal analogy and consider information and topological measures as two interpretations of one calculus in the same way classical probabilities and actual frequencies are interpretations of probability calculus, for instance. For these reasons, the question of how the concepts of entropy in these two disciplines fit together is not a trivial issue.

Critics might now ask, even if we grant that there is a problem, why we should bother with it. There are two ways to connect the KSE to unpredictability and randomness, why do we need a third one? Why not just stick with either Pessin's or Brudno's theorem? The answer to this question is that the different associations bring out different aspects of randomness that are all captured in the KSE. The connection of the KSE with communication theory adds to our understanding of these because it brings out some aspects that are particularly close to our physical intuitions, and which are not made explicit by either Pessin's or Brudno's theorem. More to the point, based on communication theoretic notions one can make statements about the time span of unpredictability or the role knowledge of the past history plays in making forecasts.

This gives rise to the following plan. After a short introduction into dy- 
namical systems (Sec. 2), I present the concept of entropy in communication theory (Sec. 3) and discuss in what sense it can be considered a measure of random or unpredictable behaviour. Although I thereby follow the spirit of Shannon and Weaver (1948), the formal presentation differs significantly from theirs. I present a version of communication theory which is in several respects a generalization of the original theory. In particular, I consider messages whose probability of appearance is a function of the entire past history of the system, while Shannon and Weaver only consider Markov processes. The purpose of this is to facilitate the establishment of the connection to entropy in dynamical systems. This is what I do in Sec. 4, where I prove an equivalence theorem for the KSE and the notion of entropy used in communication theory, this under the assumption that the measure defined on the phase space can be interpreted probabilistically. This establishes the soughtafter connection between the two notions. On the basis of this result I give a precise characterization of the kind of randomness we find in dynamical systems with positive KSE. I then compare this account to the notions of randomness we get from Pessin's or Brudno's theorems (Sec. 5). In the conclusion (Sec. 6) I point out that the main result of this paper has a bearing on the relation between product and process randomness and I argue that it casts doubt on the recent claim that chaotic systems exhibit product but not process randomness.

\section{Elements of Dynamical System Theory}

An abstract dynamical system is a triple $\mathcal{M}=\left(M, \mu, \Phi_{t}\right)$ where $(M, \mu)$ is a measure space equipped with a one-parameter group $\Phi_{t}$ of automorphisms of $(M, \mu), \Phi_{t}$ depending measurably on $t$ (Arnold and Avez 1968, 7).

It is common to assume that $M$ has manifold structure, but this is not necessary; in what follows it can be any measurable space. $M$ will be referred to as 'phase space' of the system. Sometimes this term is reserved for symplectic manifolds, but I will use it in a more general sense and refer to every measurable space on which an automorphism is defined as a 'phase space'.

The parameter $t$ plays the role of time. As it can easily be seen, the set of all $\Phi_{t}$ has group structure. In the sequel I will use the following notational conventions: $\Phi_{t}(x)$ is the point in phase space onto which $\Phi_{t}$ maps the 'initial condition' $x$ after time $t$ has elapsed, and $\Phi_{t}(A)$ is the image of the subset 
$A \subseteq M$ under $\Phi_{t}$. I write $\Phi_{t_{i} \rightarrow t_{j}}(A)$ to denote the image of $A$ under the time development starting at $t_{i}$ and ending at $t_{j}$.

Furthermore I assume, without loss of generality, that $M$ is normalized: $\mu(M)=1$. The last clause in the definition ( $\Phi_{t}$ depending measurably on $\left.t\right)$ simply means that $\Phi_{t} A \cap B$ is measurable for all $t$, and for all $A, B \in M$, i.e. that $\mu\left(\Phi_{t} A \cap B\right)$ is a measurable function of $t$. In what follows, 'almost everywhere' means 'everywhere except, perhaps, on a set of measure zero'.

As a simple example of a dynamical system in this sense think of the unit interval endowed with $\Phi_{t}$ : The shift $x \rightarrow x+t \bmod 1$ where $\mu$ is the usual Euclidean length of an interval.

The above definition is extremely general and in what follows I make the following restrictions:

(1) The transformation $\Phi_{t}$ is measure preserving: $\mu\left(\Phi_{t} A\right)=\mu(A)$ for all subsets $A$ of $M$ and all times $t$. That is, in the sequel I restrict my attention to Hamiltonian systems. Some may feel a certain unease about this limitation because they tend to think about chaos in terms of attractors, which cannot occur in Hamiltonian systems. Those may become reconciled by the following two observations. First, important paradigm cases of chaotic systems are Hamiltonian systems, for instance the three-body problem, the Hénon-Heiles system, the autonomous double pendulum, and more generally KAM-type systems. Second, apart from attractors, which are ruled out by the conservation of phase volume, Hamiltonian systems can exhibit all features that are commonly taken to be distinctive of chaotic systems: Positive Liapunov exponents, sensitive dependence on initial condition, unpredictable time evolution, continuous power spectra, decaying autocorrelations, aperiodic orbits, the presence of a stretching and folding mechanism in phase space, and last but not least positive KSE.

(2) Nothing has been said so far about whether the parameter $t$ is continuous or discrete. To keep things simple I restrict my attention to the discrete case; that is, I only consider systems in which time evolves in finite steps: $t_{1}, t_{2}, t_{3}, \ldots$. Moreover, it is often the case that the $\Phi_{t_{i}}, i=1,2, \ldots$ are generated by an iterative application of one single automorphism $\Phi$ (standard examples like the cat map or the baker's transformation belong to this class). In this case we have $\Phi_{t_{i}}=\Phi^{i}$ and $\Phi_{t_{i} \rightarrow t_{j}}(A)=\Phi^{j-i}(A)$. Furthermore, I drop the subscript and just write $(M, \mu, \Phi)$ for a dynamical system of this kind.

In what follows partitions will play an important role. Roughly, a partition of $M$ is a division of $M$ into finitely many measurable sets. More 
precisely, a partition $\alpha=\left\{\alpha_{i} \mid i=1, \ldots, n\right\}$ is a collection of non-empty, non-intersecting measurable sets that together cover $M$ : The $\alpha_{i}$ are pairwise disjoint, $\alpha_{i} \cap \alpha_{j}=\emptyset$ for all $i \neq j$; and together the $\alpha_{i}$ cover $M$ up to measure zero, $\mu\left(M-\bigcup_{i=1}^{n} \alpha_{i}\right)=0$. The $\alpha_{i}$ are called 'atoms' or 'cells' of the partition. Furthermore notice that if $\alpha$ is a partition of $M$ then its picture under the automorphism $\Phi_{t}$ is also a partition. That is, if $\alpha=\left\{\alpha_{i} \mid i=1, \ldots, n\right\}$ is a partition of $M$ then $\Phi_{t} \alpha:=\left\{\Phi_{t} \alpha_{i} \mid i=1, \ldots, n\right\}$ is as well.

Given two partitions $\alpha=\left\{\alpha_{i} \mid i=1, \ldots, n\right\}$ and $\beta=\left\{\beta_{j} \mid j=1, \ldots, m\right\}$, their least common refinement $\alpha \vee \beta$ is defined as follows: $\alpha \vee \beta=\left\{\alpha_{i} \cap \beta_{j} \mid i=\right.$ $1, \ldots, n ; j=1, \ldots, m\}$. Sometimes $\alpha \vee \beta$ is also called 'sum of $\alpha$ and $\beta$ '. Fig. 1 provides an example illustrating this.

Figure 1: The sum of the partitions $\alpha$ and $\beta$.

We are now in a position to state the definition of the Kolmogorov-Sinai entropy $H_{\Phi}$ of an automorphism $\Phi$ (Arnold and Avez 1968, 38-40):

$$
H_{\Phi}:=\sup _{\alpha} \lim _{k \rightarrow \infty}(1 / k) H\left(\alpha \vee \Phi \alpha \vee \ldots \vee \Phi^{k-1} \alpha\right),
$$

where the function on the right-hand side is the entropy of a partition (recall that $\alpha \vee \Phi \alpha \vee \ldots \vee \Phi^{k-1} \alpha$ is a partition as well) which is defined as follows: $H(\beta):=-\sum_{i=1}^{m} z\left[\mu\left(\beta_{i}\right)\right], z(x)=x \log (x)$ if $x>0$ and $z(x)=0$ if $x=0$; and $\sup _{\alpha}$ is the supremum over all possible finite partitions $\alpha$ of the phase space. I shall discuss this definition in detail later on.

\section{Entropy in Communication Theory}

Consider the following situation: We have a source $S$ producing messages which are communicated to a receiver $R$ registering them (on a paper tape, 
for instance). ${ }^{2}$ The messages may be of various types: sequences of letters, numbers, words, ... or any other symbols we might think of. The only restriction we impose is that the source uses discrete symbols and generates the message symbol by symbol. The product of this process is a string of symbols, the message, which can be of finite or infinite length.

More precisely, let $S_{1}, \ldots, S_{n}$ be the available symbols and let the process of the composition of a message start at time $t_{0}$. At that time no symbol has been produced by $S$ and the tape of $R$ is blank. The first symbol is put out by $S$ and sent to $R$ at $t_{1}$, where it is registered; the second symbol is sent and registered at $t_{2}$ (we assume that $t_{1}<t_{2}<t_{3}<\ldots$ ), and so on. The production of one symbol by the source (when it moves from $t_{i}$ to $t_{i+1}$ ) will be referred to as a 'step'. As a result of this, at $t_{k}$ the tape of $R$ contains a string of $k$ symbols: $S_{l_{1}}^{t_{1}} S_{l_{2}}^{t_{2}} \ldots S_{l_{k}}^{t_{k}}$, where all the $l_{i}$ range over $1, \ldots, n$ (i.e. the number of symbols available). The time-superscripts have been added to indicate the order of reception $\left(S_{l_{1}}\right.$ has been sent and received at $t_{1}$, and so on). For instance, assuming that our symbols are letters, $h^{t_{1}} e^{t_{2}} l^{t_{3}} l^{t_{4}} o^{t_{5}}$ means that the letter ' $h$ ' has been received at time $t_{1}$, ' $e$ ' at $t_{2}$ and so on.

I now introduce in seven stages the notion of the entropy of a source $S$, which will be designed such that it serves as a measure of the receiver's average uncertainty about what message the source produces next (that is, it is a measure for unpredictability - I shall use 'uncertainty' and 'unpredictability' interchangeably in what follows). Thereby it also is a measure of the amount of information received. I shall come to that below.

Stage 1: To start with, consider a source which has just two symbols (0 and 1 , say) it can send. What is the amount of uncertainty of the receiver about what message will crop up next? We answer this question by adopting the convention that the amount of uncertainty of the receiver in this case equals one. This is a reasonable choice: If we had just one symbol available, we in fact would know for sure that what the receiver would indicate whenever we switch it on; there is no uncertainty. The simplest non-trivial situation is the one considered here where two symbols are available. We then are not sure about what the next message will be (we could get either of the two messages) and it seems reasonable to say that the amount of uncertainty in

\footnotetext{
${ }^{2}$ In what follows I assume that the channel is noiseless and deterministic, basically meaning that there is a one-to-one correspondence between the input and the output messages.
} 
this case is one because there is one choice to be made.

Before continuing with the development of the theory I would like to make some remarks about information. It is one of the main insights of Shannon's Mathematical Theory of Communication that uncertainty is closely related to information. If we are sure about what message we receive next, we do not learn anything by actually receiving it. Therefore the amount of information transmitted is zero. If, on the other hand, there are several possibilities (e.g. if we don't know whether we will obtain 0 or 1 ), we do acquire information when we receive either of the two. For those who find this a bit contrived, consider Lewis Carroll's remark in Through the LookingGlass: 'It is a very inconvenient habit of kittens (Alice had once made the remark) that, whatever you say to them, they always purr. "If they would only purr for 'yes' and mew for 'no,' or any rule of that sort," she had said, "so that one could keep up a conversation! But how can you talk with a person if they always say the same thing?".' $(1998,238)$ In short, uncertainty about what comes next and the transmission of information are two sides of the same coin.

For this reason, devising a measure of the amount of uncertainty about future events and the quantity of information transmitted amounts to the same. Consider again the previous example. What is the amount of information transmitted when $R$ has registered ' 1 ' or ' 0 ' on its tape? For the same reasons as outlined above it is natural to say that the amount of information transmitted is one (in technical jargon, we get one 'binary digit', 'bit' for short, of information). As a consequence, when we in what follows devise entropy as a measure of the amount of uncertainty it also is a measure of the amount of information transmitted. However, the focus in the rest of the paper will be on uncertainty not on information, though I use information jargon at times if this turns out to be convenient.

Two remarks about this concept of information should be made. First, contrary to the concept of meaning, which applies to a single message (receiving ' $S_{1}$ ', for instance, could mean, 'I love you', 'I hate you', 'happy birthday' ... or what have you), information is not concerned with individual messages, but only with the ensemble of all messages a source could possibly send. What makes a single message informative is not its meaning but the fact that it is selected from a set of possible messages. The more (different) messages the source could in principle send, the higher the information content of the one we actually get. Second, from what has been said so far it is 
obvious that we are dealing here with a rather idiosyncratic concept of information which has little to do with the various senses the term 'information' has in ordinary discourse, such as knowledge or propositional content. Information in these senses has semantic features such as truth-values, something the communication-theoretic concept lacks. This has led many to criticize this concept as misconceived. Be this as it may, my focus in this paper is uncertainty and not information and for this reason I will not dwell on this issue here.

Stage 2: How does this generalize to a source which can emit $n$ different symbols? This question is best answered by looking at the restrictions we want to impose on a measure of the amount of uncertainty: (a) It must be a monotonically increasing function of $n$; the more possibilities there are, the greater our uncertainty about what comes next. (b) Additivity: When we add two sources of the same type we want the amount of uncertainty to double. Informally, let $I$ stand for uncertainty (or information). Then we require $I($ source $1+$ source 2$)=I($ source 1$)+I($ source 2$)$ (see Shannon and Weaver 1949, 32, for a justification of this assumption). (c) Finally, we should have $I=1$ in the case of only two possibilities (see Stage 1). The only function which satisfies these criteria is the logarithm to the base 2. Hence, let ' $I$ ' stand for the amount of information conveyed by $S$ per step, and ' $\log$ ' for the logarithm to the base 2 , then we have $I=\log (n)$.

Stage 3: So far we have implicitly assumed that all symbols occur with equal probability at any step $k$, i.e. that all $S_{i}$ occur with probability $p_{k}=$ $1 / n$ at step $k$ (since the probabilities $p_{k}$ do not actually depend on the step $k$, I drop the subscript $k$ in what follows). This a perfectly good assumption in certain cases, but it does not generally hold true. If, for instance, the symbols are letters of the alphabet, the probability that the next letter the sender emits is an 'a' is much higher than that for an ' $x$ ', since ' $a$ ' occurs much more often in English than ' $\mathrm{x}$ '. So we need a generalization of $I$ to this case. Let $p\left(S_{1}\right), \ldots, p\left(S_{n}\right)$ (where $p\left(S_{1}\right)+\ldots+p\left(S_{n}\right)=1$ ) be the respective probabilities that $S_{1}, \ldots, S_{n}$ occur. Shannon showed that a natural generalization of the above notion is the following (Shannon and Weaver 1949, 48-53):

$$
H_{\text {step }}:=-\sum_{i=1}^{n} z\left[p\left(S_{i}\right)\right]
$$

where $z(x)=x \log (x)$ if $x>0$ and $z(x)=0$ if $x=0 . H_{\text {step }}$ is measure of the uncertainty about what symbol will crop up at the next step; the greater $H_{\text {step }}$ 
the less certain we are about the outcome. The use of the letter ' $H$ ' instead of ' $I$ ' is motivated by the fact that Eq. (2) has the same structure as the expression for the entropy in statistical mechanics and for this same reason we also refer to it as 'entropy'. $H_{\text {step }}$ is a natural generalization of $I$ for the following reasons: First, if all events are equally probable $\left(p\left(S_{i}\right)=1 / n\right.$ for all $i=1, \ldots, n)$ it coincides with the above notion, that is $H_{\text {step }}=\log (n)$, as some simple algebra immediately reveals. Second, it is continuous in the $p\left(S_{i}\right)$. Third, it has the 'right' behaviour: (a) Any change toward equalization of the probabilities $p\left(S_{1}\right), \ldots, p\left(S_{n}\right)$ increases $H$. In particular, $H_{\text {step }}$ is maximal if all events are equally probable. (b) $H_{\text {step }}=0$ iff all $p\left(S_{i}\right)$ but one equal zero, i.e. if there is in fact no choice (ibid. 51).

Stage 4: So far nothing has been said about what the $p\left(S_{i}\right)$ are and on what they depend. In many cases the choice of a symbol at some particular time $t_{k+1}$ does not depend on previous choices. However, for a general source, the probability that a particular symbol is chosen may depend on what has been chosen beforehand. For example, if the source is producing English prose, there are a number of limitations due to the orthography and syntax of the language. The probability of receiving a ' $u$ ', for instance, will rise dramatically each time a ' $q$ ' comes through, and it will be almost zero after an ' $\mathrm{x}$ '. In short, successive symbols may not be chosen independently and their probabilities may depend on preceding letters. In the simplest case, a so-called Markov process, a choice depends only on the preceding letter and not on the ones before that. However, the choice may in general depend on the entire previous history of the process; that is, the choice of a symbol at $t_{k+1}$ may depend on $S_{l_{1}}^{t_{1}} S_{l_{2}}^{t_{2}} \ldots S_{l_{k}}^{t_{k}}$. It is natural to account for this by using conditional probabilities: The probability of receiving $S_{i}$ at time $t_{k+1}$ is $p\left(S_{i}^{t_{k+1}} / S_{l_{1}}^{t_{1}} S_{l_{2}}^{t_{2}} \ldots S_{l_{k}}^{t_{k}}\right)$. Since these probabilities may vary with $k$, the entropy may have a different value at every step. To make this explicit, I replace the subscript 'step' in Eq. (2) by ' $k$ ' to emphasise that we are considering the entropy at the $k^{\text {th }}$ step of the process. The expression for the entropy then reads:

$$
H_{k}\left(S_{l_{1}}^{t_{1}} S_{l_{2}}^{t_{2}} \ldots S_{l_{k}}^{t_{k}}\right):=-\sum_{i=1}^{n} z\left[p\left(S_{i}^{t_{k+1}} / S_{l_{1}}^{t_{1}} S_{l_{2}}^{t_{2}} \ldots S_{l_{k}}^{t_{k}}\right)\right]
$$

This is a measure for the uncertainty about what symbol will show up at time $t_{k+1}$ given that the previous history of the process (recorded on $R$ 's 
tape) is $S_{l_{1}}^{t_{1}} S_{l_{2}}^{t_{2}} \ldots S_{l_{k}}^{t_{k}}$.

Stage 5: Now we generalize our question slightly: Instead of asking 'what is the uncertainty about the $(k+1)^{t h}$ symbol given that the message produced so far is $S_{l_{1}}^{t_{1}} S_{l_{2}}^{t_{2}} \ldots S_{l_{k}}^{t_{k}}$ ?' (to which Eq. (3) is an answer) we now ask "what is the uncertainty about the $(k+1)^{t h}$ symbol whatever message has been produced so far?'. Or in other words: What is the uncertainty at $t_{k+1}$ if we do not presuppose that the system has a particular previous history, namely $S_{l_{1}}^{t_{1}} S_{l_{2}}^{t_{2}} \ldots S_{l_{k}}^{t_{k}}$ ? The answer seems clear: Take the average of all $H_{k}\left(S_{l_{1}}^{t_{1}} S_{l_{2}}^{t_{2}} \ldots S_{l_{k}}^{t_{k}}\right)$ and, to do justice to the fact that not all histories are equally likely, weight each term with the probability of the respective history:

$$
\bar{H}_{k}:=\sum_{l_{1}, \ldots, l_{k}=1}^{n} p\left(S_{l_{1}}^{t_{1}} S_{l_{2}}^{t_{2}} \ldots S_{l_{k}}^{t_{k}}\right) H_{k}\left(S_{l_{1}}^{t_{1}} S_{l_{2}}^{t_{2}} \ldots S_{l_{k}}^{t_{k}}\right)
$$

where

$$
p\left(S_{l_{1}}^{t_{1}} S_{l_{2}}^{t_{2}} \ldots S_{l_{k}}^{t_{k}}\right):=p\left(S_{l_{1}}^{t_{1}}\right) p\left(S_{l_{2}}^{t_{2}} / S_{l_{1}}^{t_{1}}\right) \ldots p\left(S_{l_{k}}^{t_{k}} / S_{l_{1}}^{t_{1}} \ldots S_{l_{k-1}}^{t_{k-1}}\right) .
$$

Stage 6: On the basis of this we can now define the entropy $\tilde{H}_{k}$ of the entire process of the composition of a message of length $k$. Since no step is privileged over the others, this can be effected by simply taking the average of the entropy at every step of the process:

$$
\tilde{H}_{k}:=\frac{1}{k} \sum_{j=0}^{k-1} \bar{H}_{j}
$$

Stage 7: Now we can say that the entropy of the source itself, $H_{S}$, is the average of the uncertainty at every step if we let the process go on forever:

$$
H_{S}:=\lim _{k \rightarrow \infty} \tilde{H}_{k}
$$

This is the so-called entropy of the source. It is a measure for the average uncertainty over the entire process or, to put it differently, the average amount of information which the source conveys with every symbol the receiver prints. I will refer to this notion of entropy as 'communicationtheoretic entropy', CTE for short. ${ }^{3}$

\footnotetext{
${ }^{3}$ Note that if we assume that all the probabilities are independent (this is the case for Bernoulli processes, for instance) we have $H_{S}=H_{\text {step }}$. This is easy to see: For independent
} 
From a technical point of view the development of the theory is now complete. But what is its conceptual import? What does it mean for a source to have a positive CTE? And in what sense is a positive CTE a measure for random behaviour? In the remainder of this section I shall discuss these questions.

Let us start by having a look at the probabilities involved. When probabilities are introduced into the theory they are assumed to be given; there is no element in the theory that determines what their values are. For this reason one could also say that the set of possible messages $S_{1}, \ldots, S_{n}$ together with the conditional probabilities of occurrence $p\left(S_{i}^{t_{k+1}} / S_{l_{1}}^{t_{1}} S_{l_{2}}^{t_{2}} \ldots S_{l_{k}}^{t_{k}}\right)$, $i=1, \ldots, n$ actually defines the source. Characteristically, these probabilities are past relative frequencies, and it is assumed that these relative frequencies will persist.

However, this 'natural order' of proceeding can in a certain sense be reversed: The entropy can be used to characterize the probabilities involved even if they are not explicitly known (leaving aside the question of how we get to know the entropy without knowing the probabilities). The point is the following. The notion of entropy has been set up in such a way that it is a measure for the average uncertainty per symbol over the entire process. For this reason, $H_{S}>0$ expresses the fact that, on average, at every step there is some uncertainty about what the next symbol printed by the receiver will be. More precisely, whatever the past history of the system and whatever our knowledge about it (we may know it all), we are not sure as to what the next symbol that emerges will be. And this characteristic persists forever, there exists no 'cut-off time' $t_{c}$ in the process from which on the past history of the system allows us to predict with certainty what its future will be. ${ }^{4}$ This follows immediately from the definition of the CTE.

events Eq. (4) becomes:

$$
\bar{H}_{k}:=-\sum_{l_{1}, \ldots, l_{k}=1}^{n} p\left(S_{l_{1}}\right) p\left(S_{l_{2}}\right) \ldots p\left(S_{l_{k}}\right) \sum_{i=1}^{n} z\left[p\left(S_{i}\right)\right],
$$

Now realize that the two sums separate and that the first one is just a sum over the probabilities of all strings of length $k$. For this reason we have: $\sum_{l_{1}, \ldots, l_{k}=1}^{n} p\left(S_{l_{1}}\right) p\left(S_{l_{2}}\right) \ldots p\left(S_{l_{k}}\right)=1$. Hence $\bar{H}_{k}(S)=-\sum_{i=1}^{n} z\left[p\left(S_{i}\right)\right]$ and therefore $H_{S}=-\sum_{i=1}^{n} z\left[p\left(S_{i}\right)\right]$.

${ }^{4}$ I should note that there is a subtle difference between "with probability equal to 
$H_{S}=\lim _{k \rightarrow \infty}(1 / k) \sum_{j=0}^{k-1} \bar{H}_{j}=\lim _{k \rightarrow \infty}\left(\bar{H}_{0} / k+\ldots+\bar{H}_{k-1} / k\right)$ is greater than zero only if there do not cease to be $\bar{H}_{k}$ greater than zero. Now recall that $\bar{H}_{k}$ is a measure for the uncertainty about what the message printed at time $k+1$ will be. Hence, if there do not cease to be $\bar{H}_{k}>0$ as time goes on, there will always be times at which we are not sure about what is going to happen. As a consequence, we cannot predict with certainty what the future will be. ${ }^{5}$ In terms of probabilities this means that as the process goes on we never reach a stage where $p\left(S_{i}^{t_{k+1}} / S_{l_{1}}^{t_{1}} S_{l_{2}}^{t_{2}} \ldots S_{l_{k}}^{t_{k}}\right)$ equals one for some particular symbol and zero for all the others.

Summing up, we can characterize a system with positive entropy as one in which the past history never conveys certainty onto what will happen at the next step and more generally in the future. Or to phrase it differently, even given the entire past history we are not able to predict with certainty what will happen in the future. It is in this sense that a process with positive entropy is random, and the magnitude of the entropy is a measure of how random it is.

If, on the other hand, $H_{S}$ equals zero, then, on average, there is no uncertainty and we can predict what the future will look like. There is a subtlety, however. Zero entropy does not imply that the process is deterministic (by which I here simply mean that given the state of a process at time $t_{k}$, there is exactly one state in which it can be at $\left.t_{k+1}\right)$. It is true that for a deterministic process $H_{S}=0$ holds. But the converse is false: $H_{S}=0$ does not imply that the process is deterministic, it just means that on average there is no freedom of choice. This does not preclude that the process is indeterministic at some particular instants of time.

\section{Entropy in Dynamical System Theory}

To repeat, the KSE of an automorphism is defined as $H_{\Phi}=\sup _{\alpha} \lim _{k \rightarrow \infty}(1 / k) H(\alpha \vee$ $\left.\Phi \alpha \vee \ldots \vee \Phi^{k-1} \alpha\right)$ and it is commonly used as a measure for the unpredictabil-

one' and 'certainty'. The latter implies the former but not vice versa. However, since this subtlety does not play any role in what follows, I shall use these two expressions interchangeably.

${ }^{5}$ This does not mean that there are no instants of time for which this is possible. As the above formula shows, $H_{S}>0$ is compatible with there being some particular $\bar{H}_{k}=0$ from time to time. The point is just that as $k$, i.e. time, goes on we never reach a point after which all $\bar{H}_{k}$ equal zero; and this is all we need to render the future unpredictable. 
ity of the dynamics. But as I explained in the introduction, it is prima facie not clear whether this is legitimate or not. In this section I show that it is by proving, under plausible assumptions, that the KSE is equivalent to the CTE.

Wanting to prove this equivalence we face the following problem: the messages we have been dealing with so far are discrete entities, whereas the phase space of a dynamical system is continuous. These two things do not seem to go together. This mismatch can be removed if we coarse grain the phase space, i.e. if we work with a partition instead of the 'whole' phase space. Then it is no longer difficult to associate a dynamical system $\mathcal{M}$ with an information source of the type discussed above. This association is achieved as follows. Let $\alpha=\left\{\alpha_{1}, \ldots, \alpha_{n}\right\}$ be a partition of the phase space and assume that the state of the system at $t_{0}$ is $x$ (more needs to be said about the choice of a partition; I come back to this issue below at stage 8). Then trace the trajectory $\Phi_{t_{i}}(x)$ of $x$ and take down on a paper tape at each time $t_{i}, i=1,2, \ldots$, in what cell $\alpha_{j}, j=1, \ldots, n$, of the partition $\Phi_{t_{i}}(x)$ is. That is, write down $\alpha_{j}^{t_{1}}$ if $\Phi_{t_{1}}(x) \in \alpha_{j}$ at time $t_{1}$ and so on. If we do that up to time $t_{k}$ this generates the string $\alpha_{l_{1}}^{t_{1}} \alpha_{l_{2}}^{t_{2}} \ldots \alpha_{l_{k}}^{t_{k}}$, which is structurally identical to $S_{l_{1}}^{t_{1}} S_{l_{2}}^{t_{2}} \ldots S_{l_{k}}^{t_{k}}$. This is illustrated in Fig. 2.

Figure 2: The generation of the string $\alpha_{8}^{t_{1}} \alpha_{1}^{t_{2}} \alpha_{2}^{t_{3}} \alpha_{9}^{t_{4}} \alpha_{16}^{t_{5}} \alpha_{18}^{t_{6}} \alpha_{6}^{t_{7}} \alpha_{10}^{t_{8}}$.

Now we need to find something in $\mathcal{M}$ corresponding to the probability $p\left(S_{i}\right)$ of choosing a particular symbol $S_{i}$. This is not too hard to get. By assumption, there is a normalised measure $\mu$ on $M$ (that is $\mu(M)=1$ ) and it is a straightforward move to interpret this measure as a probability measure. 
More precisely, let $\mu$ reflect our ignorance about the real state of the system, and interpret $\mu\left(\alpha_{i}\right)$ as the probability of finding the system's state in $\alpha_{i}$. Note, however, that although this move is quite natural, the interpretation of $\mu$ as the probability of finding the system's state in a particular cell is by no means compulsary. Not all measures reflect our ignorance about the system's real state; it could also simply be the spatial volume. However, this interpretation is perfectly possible and it allows us to connect what happens in dynamical systems to communication theory as outlined above, and that is all we need for the time being.

Then, the following associations are made to connect dynamical systems to communication theory:

(a) The atoms of the partition $\alpha_{i}$ correspond to the symbols (messages) $S_{i}$ of the source.

(b) The measures of an atom $\mu\left(\alpha_{i}\right)$, interpreted as the probability of finding the system's state in cell $\alpha_{i}$, corresponds to the probability $p\left(S_{i}\right)$ of obtaining symbol $S_{i}$.

(c) The automorphism $\Phi_{t}$ corresponds to the source $S$, since they both do the job of generating the strings $\alpha_{l_{1}}^{t_{1}} \alpha_{l_{2}}^{t_{2}} \ldots \alpha_{l_{k}}^{t_{k}}$ and $S_{l_{1}}^{t_{1}} S_{l_{2}}^{t_{2}} \ldots S_{l_{k}}^{t_{k}}$ respectively.

With these associations at hand it is now possible to carry over the notions introduced in the last section to the present context.

Stage 1: To begin with, consider a partition consisting of two atoms, $\alpha=\left\{\alpha_{1}, \alpha_{2}\right\}$, and assume that the state of the system at $t_{0}$ is $x$. Then trace the trajectory $\Phi_{t_{i}}(x)$ and take down at each time step in what cell $\alpha_{j}$, $j=1,2, \Phi_{t_{i}}(x)$ is. This generates a string of exactly the same sort as the one we obtain from a source which can send only two symbols.

As in the case of the source, we are generally not sure about what cell the system's state will be in next. Due to restrictions on the precision of the specification of initial conditions we normally cannot know precisely what the system's initial state is and this uncertainty is then propagated, or even amplified, by the dynamics of the system as time evolves. Therefore we gain information, i.e. remove uncertainty, when we learn that the system's state is in $\alpha_{1}$ rather than $\alpha_{2}$, say. Adopting the same convention as in the case of sources, we can say that the amount of uncertainty of the observer about what cell the system's state will be in at the next step is one bit.

Stage 2: Replacing this partition by one consisting of more than two atoms is the analogue to the transition from a source with two symbols to one with any number of symbols. The considerations concerning the general 
properties of information then carry over one-to-one. The more possibilities we have (i.e. the more cells the partition consists of), the greater the uncertainty about what happens next becomes. Combining two systems of the same sort should result in the doubling of the amount of uncertainty; and in the case of a partition with only two cells the uncertainty must be unity in order to be consistent with stage 1 . So, as in the case of the source, we set $I=\log (n)$.

Stage 3: By assumption, the measure $\mu\left(\alpha_{j}\right)$ is interpreted as the probability that at the next step the system will be in cell $\alpha_{j}$; that is, we have $p\left(\alpha_{j}\right)=\mu\left(\alpha_{j}\right)$. This allows us to carry over Eq.(2) to the present context. We immediately obtain $H_{\text {step }}=-\sum_{i=1}^{n} z\left[\mu\left(\alpha_{i}\right)\right]$, which is commonly called the 'entropy of the partition $\alpha$ '. To be in accord with the notation commonly used in the literature I write ' $H(\alpha)$ ' instead of ' $H_{\text {step }}$ ':

$$
H(\alpha):=-\sum_{i=1}^{n} z\left[\mu\left(\alpha_{i}\right)\right]
$$

Stage 4: In general, also in dynamical systems the previous history affects future probabilities. Therefore Eq. (3) carries over to the present context:

$$
H_{k}\left(\alpha ; \alpha_{l_{1}}^{t_{1}} \alpha_{l_{2}}^{t_{2}} \ldots \alpha_{l_{k}}^{t_{k}}\right):=-\sum_{i=1}^{n} z\left[p\left(\alpha_{i}^{t_{k+1}} / \alpha_{l_{1}}^{t_{1}} \alpha_{l_{2}}^{t_{2}} \ldots \alpha_{l_{k}}^{t_{k}}\right)\right],
$$

Now we have to express the probabilities occurring in this expression in terms of $\mu$. To this end, first spell out the conditional probability in terms of unconditional ones:

$$
\begin{aligned}
p\left(\alpha_{i}^{t_{k+1}} / \alpha_{l_{1}}^{t_{1}} \alpha_{l_{2}}^{t_{2}} \ldots \alpha_{l_{k}}^{t_{k}}\right) & =p\left(\alpha_{i}^{t_{k+1}} \& \alpha_{l_{1}}^{t_{1}} \alpha_{l_{2}}^{t_{2}} \ldots \alpha_{l_{k}}^{t_{k}}\right) / p\left(\alpha_{l_{1}}^{t_{1}} \alpha_{l_{2}}^{t_{2}} \ldots \alpha_{l_{k}}^{t_{k}}\right) \\
& =p\left(\alpha_{l_{1}}^{t_{1}} \alpha_{l_{2}}^{t_{2}} \ldots \alpha_{l_{k}}^{t_{k}} \alpha_{i}^{t_{k+1}}\right) / p\left(\alpha_{l_{1}}^{t_{1}} \alpha_{l_{2}}^{t_{2}} \ldots \alpha_{l_{k}}^{t_{k}}\right)
\end{aligned}
$$

The latter equality follows immediately from the definition of a string.

Realize that for any two instants of time $t_{i}$ and $t_{j}$ (where $t_{i}<t_{j}$ ) and any two subsets $A$ and $B$ of $M$ the following holds:

$$
p\left(A^{t_{i}} B^{t_{j}}\right)=\mu\left[\Phi_{t_{i} \rightarrow t_{j}}(A) \cap B\right] .
$$

The validity of this equation becomes transparent from Fig. 3.

The generalisation of this equality to any number of sets and instants of time is straightforward. Applying this to the above expressions yields:

$$
p\left(\alpha_{l_{1}}^{t_{1}} \alpha_{l_{2}}^{t_{2}} \ldots \alpha_{l_{k}}^{t_{k}}\right)=\mu\left(\alpha_{l_{k}} \cap \Phi_{t_{k-1} \rightarrow t_{k}} \alpha_{l_{k-1}} \cap \ldots \cap \Phi_{t_{1} \rightarrow t_{k}} \alpha_{l_{1}}\right),
$$


and

$$
p\left(\alpha_{l_{1}}^{t_{1}} \alpha_{l_{2}}^{t_{2}} \ldots \alpha_{l_{k}}^{t_{k}} \alpha_{i}^{t_{k+1}}\right)=\mu\left(\alpha_{i} \cap \Phi_{t_{k} \rightarrow t_{k+1}} \alpha_{l_{k}} \cap \ldots \cap \Phi_{t_{1} \rightarrow t_{k+1}} \alpha_{l_{1}}\right) .
$$

Hence (10) becomes:

$$
H_{k}\left(\alpha ; \alpha_{l_{1}}^{t_{1}} \alpha_{l_{2}}^{t_{2}} \ldots \alpha_{l_{k}}^{t_{k}}\right):=-\sum_{i=1}^{n} z\left[\frac{\mu\left(\alpha_{i} \cap \Phi_{t_{k} \rightarrow t_{k+1}} \alpha_{l_{k}} \cap \ldots \cap \Phi_{t_{1} \rightarrow t_{k+1}} \alpha_{l_{1}}\right)}{\mu\left(\alpha_{l_{k}} \cap \Phi_{t_{k-1} \rightarrow t_{k}} \alpha_{l_{k-1}} \cap \ldots \cap \Phi_{t_{1} \rightarrow t_{k}} \alpha_{l_{1}}\right)}\right] .
$$

Figure 3: The probability of $A^{t_{i}} B^{t_{j}}$ equals $\mu\left[\Phi_{t_{i} \rightarrow t_{j}}(A) \cap B\right]$.

Stage 5: Similarly, Eq. (4) carries over to dynamical systems easily:

$$
\bar{H}_{k}(\alpha):=\sum_{l_{1}, \ldots, l_{k}=1}^{n} p\left(\alpha_{l_{1}}^{t_{1}} \alpha_{l_{2}}^{t_{2}} \ldots \alpha_{l_{k}}^{t_{k}}\right) H_{k}\left(\alpha_{l_{1}}^{t_{1}} \alpha_{l_{2}}^{t_{2}} \ldots \alpha_{l_{k}}^{t_{k}}\right)
$$

which is the entropy of the $k^{t h}$ step relative to the partition $\alpha$. Inserting probabilities in terms of the measures we obtain

$$
\begin{aligned}
\bar{H}_{k}(\alpha):= & \sum_{l_{1}, \ldots, l_{k}=1}^{n} \mu\left(\alpha_{l_{k}} \cap \Phi_{t_{k-1} \rightarrow t_{k}} \alpha_{l_{k-1}} \cap \ldots \cap \Phi_{t_{1} \rightarrow t_{k}} \alpha_{l_{1}}\right) \\
& \sum_{i=1}^{n} z\left[\frac{\mu\left(\alpha_{i} \cap \Phi_{t_{k} \rightarrow t_{k+1}} \alpha_{l_{k}} \cap \ldots \cap \Phi_{t_{1} \rightarrow t_{k+1}} \alpha_{l_{1}}\right)}{\mu\left(\alpha_{l_{k}} \cap \Phi_{t_{k-1} \rightarrow t_{k}} \alpha_{l_{k-1}} \cap \ldots \cap \Phi_{t_{1} \rightarrow t_{k}} \alpha_{l_{1}}\right)}\right] .
\end{aligned}
$$

Stage 6: The entropy of the process of the composition of a string of length $k$ is: 


$$
\tilde{H}_{k}(\alpha):=\frac{1}{k} \sum_{j=0}^{k-1} \bar{H}_{j}(\alpha) .
$$

Stage 7: On the basis of this we define the entropy of an automorphism as follows:

$$
H_{\Phi_{t}}(\alpha):=\lim _{k \rightarrow \infty} \tilde{H}_{k}(\alpha),
$$

This is the entropy of the automorphism $\Phi_{t}$ with respect to the partition $\alpha$.

Stage 8: At the beginning of this section I mentioned in passing that more needs to be said about the choice of a partition. The reason for this is that there is an important disanalogy between a source and a dynamical system. In the case of a Source $S$, the set of possible messages $\left(S_{1}, \ldots, S_{n}\right)$ is a part of the definition of the source and hence it is no longer an issue later on. This is not so with the partition $\alpha$, which is no constitutive part of the dynamical system $\mathcal{M}$. Rather it has been 'imposed' on the system.

This is a problem because the values we obtain for $H_{\Phi_{t}}(\alpha)$ essentially depend on the choice of the partition $\alpha$. If we choose $\alpha$ conveniently enough (which we always can, no restrictions having been imposed on $\alpha$ ), there will be no uncertainty left, whatever the properties of $\Phi_{t}$ (this can be achieved, for instance, by choosing the trivial partition, i.e. the partition whose only atom is $M$ itself). Hence, prima facie $H_{\Phi_{t}}(\alpha)$ tells us more about our choice of $\alpha$ than about the properties of the automorphism $\Phi_{t}$.

This may pose no problem if the partition $\alpha$ is what we are ultimately interested in. But for the most part we are interested in the automorphism $\Phi_{t}$ itself rather than the partition, which merely is an auxiliary device. For this reason we have to eliminate the dependence on $\alpha$ and get to a notion of entropy which does no longer dependent on any particular partition.

This can be achieved by defining the entropy of the automorphism as the supremum of $H_{\Phi_{t}}(\alpha)$ over all finite measurable partitions (Arnold and Avez 1968, 40):

$$
H_{\Phi_{t}}=\sup _{\alpha} H_{\Phi_{t}}(\alpha) .
$$

The choice of the supremum is motivated by the following considerations. From the point of view of the dynamical system there is no privileged partition, one is just as good as any other. Therefore it is interesting to discuss how $H_{\Phi_{t}}(\alpha)$ behaves as a function of $\alpha$, when $\alpha$ ranges over all finite measurable partitions. As I just observed, one can always find a partition such 
that $H_{\Phi_{t}}(\alpha)=0$; and from the definition of $H_{\Phi_{t}}(\alpha)$ it follows that it cannot be negative. Hence zero is a infimum of $H_{\Phi_{t}}(\alpha)$. However, This is not a very informative result if we want to know something about the automorphism $\Phi_{t}$, since this holds true regardless of what $\Phi_{t}$ is. So what about the supremum? This is an interesting question because the supremum really depends on $\Phi_{t}$. Some automorphisms are so such that we simply cannot find a partition with respect to which there is much uncertainty, while with others things get as unpredictable as we may want. For this reason the supremum of $H_{\Phi_{t}}(\alpha)$, unlike the infimum, tells us a great deal about the automorphism. More specifically, it informs us about the maximal magnitude of uncertainty we can encounter in a system governed by $\Phi_{t}$.

But there is a problem: The expression in Eq. (21) does not bear any resemblance whatsoever to the standard definition of the KSE. I now solve this problem by proving that, $H_{\Phi_{t}}$ as defined above, is equivalent to the standard definition.

To this end, I first have to introduce a technical device, the so-called conditional entropy. Let $\alpha$ and $\beta$ be two partitions. The conditional entropy of $\alpha$ with respect to $\beta$ is defined as follows (Arnold and Avez 1968, 37):

$$
H(\alpha / \beta):=\sum_{j=1}^{m} \mu\left(\beta_{j}\right) \sum_{i=1}^{n} z\left[\frac{\mu\left(\alpha_{i} \cap \beta_{j}\right)}{\mu\left(\beta_{j}\right)}\right]
$$

Then realize that the standard definition of the KSE assumes that the flow is generated by the iterative application of the same automorphism $\Phi$. So we have $\Phi_{t_{i}}=\Phi^{i}$ and $\Phi_{t_{i} \rightarrow t_{j}}(A)=\Phi^{j-i}(A)$ (see Sec. 2). Given this, I prove in the Appendix

THEOREM 1

$$
\bar{H}_{k}(\alpha)=H\left(\alpha / \Phi \alpha \vee \Phi^{2} \alpha \vee \ldots \vee \Phi^{k} \alpha\right) .
$$

Then the entropy of the process as given in Eq. (19) reads:

$$
\tilde{H}_{k}(\alpha)=\frac{1}{k}\left[H(\alpha)+H(\alpha / \Phi \alpha)+\ldots+H\left(\alpha / \Phi \alpha \vee \ldots \vee \Phi^{k-1} \alpha\right)\right]
$$

This can be considerably facilitated by using

\section{THEOREM 2}

$$
H\left(\alpha \vee \Phi \alpha \vee \ldots \vee \Phi^{k} \alpha\right)=H(\alpha)+H(\alpha / \Phi \alpha)+\ldots+H\left(\alpha / \Phi \alpha \vee \ldots \vee \Phi^{k} \alpha\right)
$$


Hence,

$$
\tilde{H}_{k}(\alpha)=\frac{1}{k} H\left(\alpha \vee \Phi \alpha \vee \ldots \vee \Phi^{k-1} \alpha\right)
$$

Inserting this first into (20) and then (21) we obtain

$$
H_{\Phi}=\sup _{\alpha} \lim _{k \rightarrow \infty}(1 / k) H\left(\alpha \vee \Phi \alpha \vee \ldots \vee \Phi^{k-1} \alpha\right),
$$

and this is the definition of the entropy of an automorphism towards which we were aiming. Gathering the pieces together, we have proven the

Equivalence Theorem

$$
\begin{aligned}
H_{\Phi}= & \sup _{\alpha} \lim _{k \rightarrow \infty}(1 / k) H\left(\alpha \vee \Phi \alpha \vee \ldots \vee \Phi^{k-1} \alpha\right) \\
= & \sup _{\alpha} \lim _{k \rightarrow \infty} \frac{-1}{k} \sum_{j=0}^{k-1} \sum_{l_{1}, \ldots, l_{k}=1}^{n} p\left(\alpha_{l_{1}}^{t_{1}} \alpha_{l_{2}}^{t_{2}} \ldots \alpha_{l_{k}}^{t_{k}}\right) \\
& \sum_{i=1}^{n} z\left[p\left(\alpha_{i}^{t_{k+1}} / \alpha_{l_{1}}^{t_{1}} \alpha_{l_{2}}^{t_{2}} \ldots \alpha_{l_{k}}^{t_{k}}\right)\right] .
\end{aligned}
$$

Since, by construction, the last term in this equation is equivalent to the CTE, the sought-after connection between the notion of entropy in dynamical system theory and in information theory is established.

As a consequence, everything that has been said at the end of Sec. 3 about the unpredictable behaviour of a source can be carried over to dynamical systems one-to-one. However, a proviso with regard to the choice of a partition must be made. The exact analogue of the CTE is $H_{\Phi}(\alpha)$ and not $H_{\Phi}$, which is defined as the supremum of $H_{\Phi}(\alpha)$ over all partitions $\alpha$. For this reason, the characterization of randomness devised in the context of communication theory strictly speaking applies to $H_{\Phi}(\alpha)$ rather than $H_{\Phi}$. However, there is a close connection between the two: Whenever $H_{\Phi}>0$, there trivially is at least one partition for which $H_{\Phi}(\alpha)>0$. In this case, $\Phi_{t}$ is random in precisely the way described above with respect to this partition, and more generally with respect to all partitions for which $H_{\Phi_{t}}(\alpha)>0$. For this reason, statements about $H_{\Phi}$ and $H_{\Phi}(\alpha)$ naturally translate into one another.

This said, we obtain the following characterization: If an automorphism has positive KSE then, whatever the past history of the system, we are on 
average not able to predict with certainty in what cell of the partition the system state will lie next. And this will be the case forever: There is no 'cut-off time' after which we have gathered enough information to predict what will happen in the entire future. We can collect as much knowledge about the system's past as we like and we are still left uncertain about its future. On average, we are just never sure about what happens next, since the past history does not convey certainty onto what will happen in the future (however, we may be certain of what happens at the next step at some isolated instants of time). For short, the past history does not determine the future.

Moreover, the magnitude of the KS entropy is a measure for how great our failure to predict the future will be; the greater the entropy the more uncertain we are about the future.

From this it follows immediately that the dynamics obeys the so-called 0-1 law of probability theory. This law states that even if we have complete knowledge of the process' behaviour in the past, the only events which we can predict with certainty at the next step are those which have either probability 0 or 1 independently of the past history (see Batterman 1993, 60).

\section{Comparison With Other Accounts}

In the last section I have presented a discussion of the unpredictability we find in systems with positive KSE. This characterization is, to a large extent at least, not new (similar characterizations, though less detailed, can be found in Earman 1986, Ch. 9, or Batterman 1993, Sec. 3). However, it is only the link between the KSE and the information theoretic notion of entropy which allows for a justification of this characterization. In other words, it is the result obtained in the previous section that puts this characterization on firm ground. In this section I briefly show why this is so.

As I have mentioned at the beginning, there are two other methods to link the KSE with unpredictability or randomness: (1) Use Pessins theorem which relates the KSE to positive Lyapunov exponents or (2) invoke Brudno's theorem which connects it to algorithmic complexity. I will now briefly discuss these options and explain where the differences between these and an approach based on the CTE lie.

(1) Lyapunov exponents: Sensitive dependence on initial conditions is a 
distinguishing feature of chaotic behaviour. Initially arbitrarily close points in the phase space produce markedly different trajectories. For this reason, the slightest vagueness in the specification of the initial state renders long term predictions impossible because two initially indistinguishable states will evolve into two distinguishable ones. Characteristically, trajectories in chaotic systems diverge exponentially and Lyapunov exponents (LE) proved a good quantitative measure for the average rate of exponential divergence of two trajecotries. Hence, positive LE are indicative of unpredictable behaviour. For this reason it is desirable to link the KSE to positive LE. And this is what Pessin's theorem achieves by stating that $H(\Phi)$ is basically equal to the sum of the positive LE of the system (see for instance Eckmann and Ruelle 1985, 394, or Lichtenberg and Liebermann 1991, 304).

This is a valid argument. But it does not take us as far as we can go. Nothing is said about the time span of the unpredictablity (will it last forever?), nothing has been said about how quickly predictions break down (after one time step? after two? ... after ten?), and no mention of the past history is made (how does knowledge of the past history influence our predictive abilities?). But these are of great interest in a physics context.

(2) Algorithmic complexity: An important account defines randomness in terms of algorithmic complexity. Roughly speaking, the algorithmic complexity (AC) of a sequence (here $\alpha_{l_{1}}^{t_{1}} \alpha_{l_{2}}^{t_{2}} \ldots \alpha_{l_{k}}^{t_{k}}$ ) is the length of the shortest computer program we have to provide in order to get a universal Turing machine to reproduce (compute) the sequence. We then define a sequence to be random if the shortest program of this sort has essentially the length of the sequence itself (that is, the program basically says 'print $\alpha_{l_{1}}^{t_{1}} \alpha_{l_{2}}^{t_{2}} \ldots \alpha_{l_{k}}^{t_{k}}$ '). (For details see Cover and Thomas 1991, Ch. 7; summaries can be found in Batterman 1993, Sec. 4; Belot and Earman 1997, Sec. 2; and Earman 1986, Ch. 8).

This notion of randomness can be connected to the KSE by invoking Brudno's theorem which states that for almost all trajectories (i.e. sequences $\alpha_{l_{1}}^{t_{1}} \alpha_{l_{2}}^{t_{2}} \ldots \alpha_{l_{k}}^{t_{k}}$ ) the AC of the trajectory equals the KSE of the system (Brudno 1978; for discussions see Alekseev and Yakobson 1981, Batterman and White 1996). Hence we can say that the KSE is a measure of random behaviour in the sense AC.

This is a very elegant way to interpret the KSE in terms of randomness. But is it really what we need? I think that this account is less attractive than it initially appears. The term 'randomness' may refer to many different 
things in different contexts and it is beyond the scope of this paper to discuss the variety of options. However, in the context of dynamical systems, what we mean by 'random behaviour' is unpredictable behaviour. At the most basic level, we say that an event is random if there is no way to predict its occurrence with certainty. Likewise, a random process is one for which we are not able to predict what happens next. That is, what we have in mind when we call the behaviour of a dynamical system 'random' is our inability to predict its future behaviour, and any definition of randomness we employ in this context must somehow do justice to this intuition. But this is not the case with $\mathrm{AC}$. It does not make reference to prediction and it is not clear how a connection between $\mathrm{AC}$ and predictability might be established since it is concerned only with the reproduction of a previously given sequence.

\section{Conclusion}

I would like to conclude this paper by discussing a consequence of the above result for the notion of randomness characteristic of chaotic systems. Two basic types of randomness have been distinguished, namely process and product randomness (see Earman 1986, 137-8). First, we are faced with process randomness (also referred to as genesis randomness) if we are faced with a process which operates without a hard and fast principle. A process involving genuine chance, for instance, belongs to this category. Second, the output of a process exhibits product randomness (also called performance randomness) if it is lacking a discernible pattern or if it simply is 'out of shape' in some sense or another. It is clear that product randomness is not an absolute concept. Like in the case of simplicity, we have strong and pervasive intuitions, but it is difficult to cash out in an objective sense what these amount to; what we consider as patternless depends (to some extent at least) on our point of view.

These two notions of randomness do not generally coincide. On the one hand, a sequence that is random in the product sense need not necessarily be the output of a genuinely random process. So-called 'random number generators' in digital computers are a point in case. They are programs that are set up in a way that the sequences they produce look random, but all the program performs are simple arithmetical manipulations of numbers which do not involve any stochastic element. On the other hand, process randomness 
is no sure guarantee for performance randomness, though it leads to strong expectation of a random product. It is in principle possible that a random process accidentally produces a highly ordered sequence. For instance, it is possible that if we flip a coin 1000 times we obtain 1000 heads.

For this reason it is interesting to notice that in the case of a system with positive KSE the extensions of these two notions coincide as a consequence of the above theorem. The argument runs as follows: First, AC is commonly taken to be a notion of product randomness, because it defines randomness in terms of the computational power needed to reproduce a given sequence. Second, my discussion of the CTE shows that it is a notion of process randomness: The focus is on the process in that we ask at every step what the uncertainty about the next step is. Third, Brudno's theorem states that the $\mathrm{KSE}$ is equivalent to the $\mathrm{AC}$. The above theorem states that the CTE is equivalent to the KSE. Hence, AC is equivalent to the CTE. The punch line of this is that the last equivalence equates notions of process and product randomness. This means that whenever a dynamical system behaves randomly in a process sense (cashed out in terms of CTE) then its trajectories exhibit product randomness (in the sense $\mathrm{AC}$ ), and vice versa. In short, product and process randomness are extensionally equivalent.

This has a bearing on the type of randomness we find in chaotic systems. It has been claimed recently, for instance that chaotic systems exhibit only product but not process randomness: "If there is to be randomness in chaotic models, it must be randomness in the product sense - since, by hypothesis, we are there dealing with models with thoroughly deterministic dynamics (the "processes" are entirely non-random).' (Smith 1998, 149) However, if we grant that K-systems are chaotic ${ }^{6}$, then this casts doubt on this claim since K-systems exhibit both product and process randomness.

Proponents of the argument in question might now counter that the underlying dynamics is thoroughly deterministic and for this reason there cannot be any process randomness at the very 'basic level'. True, at the level of 'mathematical' trajectories and exact initial conditions there is no ran-

\footnotetext{
${ }^{6} \mathrm{I}$ am somewhat cautious here because though I take it that being a K-system is sufficient for chaos, it is clearly not necessary; that is, not all chaotic systems are Ksystems. KAM-type systems, for instance, exhibit chaotic beaviour but they are not K-systems. In fact, they are not even ergodic due to the presence of invariant tori in the phase space. Furthermore, dissipative systems, to which the notion of being a K-system as defined in this paper does not apply, clearly can be chaotic.
} 
domness. But this reply is besides the point: Chaos and randomness only become an issue in dynamical systems once the dynamics is discussed at the coarse-grained level; as long as we assume that unlimited precision is available, there is no unpredictability or any other symptom of chaos. But once we go to the coarse-grained level, then the system exhibits both, product and process randomness.

\section{Acknowledgements}

Jossi Berkovitz, Robert Bishop, Nancy Cartwright, Jean-Michel Delhotel, Carl Hoefer, Fred Kronz, Samuel Kutter, Chuang Liu, Michael Redhead, Orly Shenker and Max Steuer have read earlier drafts of the paper and their comments were of great help to me.

\section{Appendix: Proofs of Theorems 1 and 2.}

In order to prove the two main theorems five lemmas are needed. The proof of Lemmas 1 and 3 can be found in Arnold and Avez $(1968,38)$, the other proofs are trivial.

LemMA 1: $H(\alpha \vee \beta)=H(\alpha)+H(\beta / \alpha)$.

LEMMA 2: $H(\alpha)=H\left(\Phi_{t} \alpha\right)$

Lemma 3: $\Phi(\alpha \vee \beta)=\Phi \alpha \vee \Phi \beta$.

LeMma 4: $H(\alpha \vee \beta)=H(\beta \vee \alpha)$.

LEMmA 5: $\vee$ is associative: $\alpha \vee \beta \vee \gamma=(\alpha \vee \beta) \vee \gamma=\alpha \vee(\beta \vee \gamma)$.

Proof of THEOREM 1:

For the case of an automorphism generated by a mapping we have $\Phi_{t_{i} \rightarrow t_{j}}(A)=\Phi^{j-i}(A)$ (see above). Then (18) becomes: 


$$
\bar{H}_{k}(\alpha)=-\sum_{l_{1}, \ldots, l_{k}=1}^{n} \mu\left(\alpha_{l_{k}} \cap \ldots \cap \Phi^{k-1} \alpha_{l_{1}}\right) \sum_{i=1}^{n} z\left[\frac{\mu\left(\alpha_{i} \cap \Phi \alpha_{l_{k}} \cap \ldots \cap \Phi^{k} \alpha_{l_{1}}\right)}{\mu\left(\alpha_{l_{k}} \cap \ldots \cap \Phi^{k-1} \alpha_{l_{1}}\right)}\right],
$$

Using the fact that $\Phi$ is area preserving we get $\mu\left(\alpha_{l_{k}} \cap \ldots \cap \Phi^{k-1} \alpha_{l_{1}}\right)=$ $\mu\left(\Phi \alpha_{l_{k}} \cap \ldots \cap \Phi^{k} \alpha_{l_{1}}\right)$. Plugging this into Eq. (29) and taking the associativity of set intersection into account we obtain:

$$
\bar{H}_{k}(\alpha)=-\sum_{l_{1}, \ldots, l_{k}=1}^{n} \mu\left(\Phi \alpha_{l_{k}} \cap \ldots \cap \Phi^{k} \alpha_{l_{1}}\right) \sum_{i=1}^{n} z\left[\frac{\mu\left(\alpha_{i} \cap\left\{\Phi \alpha_{l_{k}} \cap \ldots \cap \Phi^{k} \alpha_{l_{1}}\right\}\right)}{\mu\left(\Phi \alpha_{l_{k}} \cap \ldots \cap \Phi^{k} \alpha_{l_{1}}\right)}\right],
$$

Now realize that what the first sum effectively does is sum over all elements of a partition consisting of all intersections $\Phi \alpha_{l_{k}} \cap \ldots \cap \Phi^{k} \alpha_{l_{1}}$. This partition, however, is just $\Phi \alpha \vee \ldots \vee \Phi^{k} \alpha$. Furthermore, compare Eq. (30) with the definition of the conditional entropy in Eq. (22). We then obtain: $\bar{H}_{k}(\alpha)=H\left(\alpha / \Phi \alpha \vee \ldots \vee \Phi^{k} \alpha\right)$. QED.

Proof of THEOREM 2 by weak induction on $k$ :

Base case:

$H(\alpha \vee \Phi \alpha)=H(\alpha)+H(\alpha / \Phi \alpha)$.

Proof:

$H(\alpha \vee \Phi \alpha)=H(\Phi \alpha \vee \alpha)$, by Lemma 4, and $H(\Phi \alpha \vee \alpha)=H(\Phi \alpha)+H(\alpha / \Phi \alpha)$ by Lemma 1. Now use Lemma 2 and get $H(\Phi \alpha)+H(\alpha / \Phi \alpha)=H(\alpha)+$ $H(\alpha / \Phi \alpha)$. QED.

Inductive step:

$H\left(\alpha \vee \Phi \alpha \vee \ldots \vee \Phi^{k+1} \alpha\right)=H(\alpha)+\ldots+H\left(\alpha / \Phi \alpha \vee \ldots \vee \Phi^{k+1} \alpha\right)$

Proof:

Consider $H\left(\alpha \vee \Phi \alpha \vee \ldots \vee \Phi^{k+1} \alpha\right)$. With Lemmas 5 and 4 this is $H([\Phi \alpha \vee$ $\left.\left.\ldots \vee \Phi^{k+1} \alpha\right] \vee \alpha\right)$, and now applying Lemma 1 yields $H\left(\Phi \alpha \vee \ldots \vee \Phi^{k+1} \alpha\right)+$ $H\left(\alpha /\left[\Phi \alpha \vee \ldots \vee \Phi^{k+1} \alpha\right]\right)$. Lemmas 2 and 3 together with the fact that $\Phi$ is measure preserving give: $H\left(\alpha \vee \ldots \vee \Phi^{k} \alpha\right)+H\left(\alpha /\left[\Phi \alpha \vee \ldots \vee \Phi^{k+1} \alpha\right]\right)$. With the induction hypothesis this is $H\left(\alpha \vee \Phi \alpha \vee \ldots \vee \Phi^{k+1} \alpha\right)=H(\alpha)+\ldots+H(\alpha /[\Phi \alpha \vee$ $\left.\ldots \vee \Phi^{k+1} \alpha\right]$ ). QED. 


\section{References}

Alekseev, V. M. and M. V. Yakobson (1981): Symbolic Dynamics and Hyperbolic Dynamical Systems. Physics Reports 75, 287-325.

Arnold, V. I. and A. Avez (1968): Ergodic Problems of Classical Mechanics. New York.

Batterman, Robert (1993): Defining Chaos. Philosophy of Science 60, 43-66.

- and Homer White (1996): Chaos and Algorithmic Complexity. Foundations of Physics 26, 307-336.

Belot, Gordon and John Earman (1997): Chaos out of Order: Quantum Mechanics, the Correspondence Principle and Chaos. Studies in the History and Philosophy of Modern Physics 28, 147-82.

Billingsley, Patrick (1965): Ergodic Theory and Information. New York.

Brudno, A. A. (1978): The Complexity of the Trajectory of a Dynamical System. Russian Mathematical Surveys 33, 197-98.

Carroll, Lewis (1998): Alice's Adventures in Wonderland and Trough the Looking-Glass. London.

Cornfeld, I. P., S. V. Fomin, and Y. G. Sinai (1982): Ergodic Theory. Berlin, Heidelberg, New York a.o.

Cover, Thomas M. and Joy M. Thomas (1991): Elements of Information Theory. New York, Chichester a.o.

Earman, John (1986): A Primer on Determinism. Dordrecht.

Keller, Gerhard (1998): Equilibrium States in Ergodic Theory. Cambridge.

Lichtenberg A. J. and Liebermann, M. A. (1992): Regular and Chaotic Dynamics. $2^{\text {nd }}$ ed. Berlin, Heidelberg, New York a.o. 
Mañé, Ricardo (1983): Ergodic Theory and Differentiable Dynamics. Berlin, Heidelberg, New York a.o.

Nadkarni, Mahendra G. (1998): Basic Ergodic Theory. Basel.

Ott, Edward (1993): Chaos in Dynamical Systems. Cambridge.

Parry, William (1981): Topics in Ergodic Theory. Cambridge.

Rudolph, Daniel J. (1990): Fundamentals of Measurable Dynamics. Ergodic Theory on Lebesgue Spaces. Oxford.

Reichl, Linda E. (1992): The Transition to Chaos. In Conservative Classical Systems: Quantum Manifestations. New York, Berlin a.o.

Schuster, Heinz Georg (1988): Deterministic Chaos: An Introduction. $2^{\text {nd }}$ ed., Weinheim.

Shannon, Claude E. and Warren Weaver (1949): The Mathematical Theory of Communication. Urbana, Chicago and London.

Smith, Peter (1998): Explaining Chaos. Cambridge.

Sinai, Y. G. (ed.) (1980): Dynamical Systems II. Ergodic Theory with Applications to Dynamical Systems and Statistical Mechanics. Berlin, Heidelberg, New York a.o.

Tabor, Micheal (1989): Chaos and Integrability in Nonlinear Dynamics. New York a.o. 